\title{
Los escarabajos de corteza en Chile: una revisión de la situación actual e implicancias en el comercio internacional
}

\author{
Bark beetles in Chile: a review of the current situation and its effects \\ in the international trade
}

\author{
Cecilia Ruiz G ${ }^{a^{*}}$ y Dolly Lanfranco $L^{a}$ \\ *Autor de correspondencia: aUniversidad Austral de Chile, Facultad de Ciencias Forestales, Instituto de Silvicultura, \\ Valdivia, Chile, tel.: 56-63-293085, fax: 56-63-221230, ceciliaruiz@uach.cl
}

\begin{abstract}
SUMMARY
One of the main Chilean forestry exported products, and also showing better development prospects, is the saw wood of Pinus radiata; this is due to high levels of future availability of good quality logs to supply its industry and the growing global demand for this product. However, these exports have been affected by the presence, in the country, of three bark beetles introduced species: Hylurgus ligniperda, Hylastes ater and Orthotomicus erosus (Coleoptera: Scolytidae). As these insects are considered quarantine pests to major markets importing Chilean products, particularly saw wood, its presence in shipments from Chile could restrict the marketing of these products, thus producing a great impact on the regional and national economy. In 2006, the Servicio Agrícola y Ganadero (SAG) and private managers associated to the Corporación Chilena de la Madera (CORMA) suspended the green sawed wooden exports to Mexico, owing to the presence of adults of H. ligniperda in some shipments, what generated both from the point of view of the State and from the exporters as well, the implementation of some measures to correct these effects in the commercialization of these products. Some biological and ecological bark beetle aspects, its role transporting fungal spores of sapstains and pathogens, as well as its importance as quarantine pests feasible to be introduced into the main markets were analyzed and discussed. Some measures adopted by the government and the forestry sector to get to external markets with minimum risk of pests are also presented.
\end{abstract}

Key words: saw wood, bark beetle, Pinus radiata, quarantine.

\section{RESUMEN}

Uno de los principales productos forestales chilenos exportados, y además con mejores perspectivas de desarrollo, es la madera aserrada de Pinus radiata, debido a los altos índices de disponibilidad futura de trozas de buena calidad para abastecer su industria y a la creciente demanda a nivel mundial por este producto. Sin embargo, estas exportaciones se han visto afectadas por la presencia en el país de tres especies introducidas de escarabajos de la corteza: Hylurgus ligniperda, Hylastes ater y Orthotomicus erosus (Coleoptera: Scolytidae). Por tratarse de insectos considerados plagas cuarentenarias para los principales mercados que importan productos chilenos, y en este caso la madera aserrada, su presencia en embarques procedentes de Chile podría restringir la comercialización de estos productos causando un impacto en la economía regional y nacional. El año 2006 el Servicio Agrícola y Ganadero (SAG) y empresarios privados asociados en la Corporación Chilena de la Madera (CORMA) suspendieron las exportaciones de madera aserrada verde a México, por la presencia de adultos de H. ligniperda en algunos embarques. Ello generó que tanto el Estado como los exportadores implementaran algunas medidas para corregir tales situaciones en la comercialización de dichos productos. Se analizan y discuten algunos aspectos biológicos y ecológicos de los escarabajos, su rol como transportadores de esporas de hongos manchadores y patógenos, así como su importancia como plagas cuarentenarias factibles de introducirse en los principales mercados de exportación. Se presentan también las medidas adoptadas por el gobierno y las empresas del sector forestal para llegar a los mercados internacionales con riesgo mínimo de plagas.

Palabras clave: madera aserrada, escarabajos de corteza, Pinus radiata, cuarentenario.

\section{LOS ESCARABAJOS DE LA CORTEZA Y ALGUNOS ASPECTOS DE SU BIOLOGÍA EN CHILE}

A principios de la década de los 80 y posiblemente en embalajes de madera procedentes de Europa se introduje- ron al país tres especies de escolítidos barrenadores de la corteza asociados a árboles debilitados, tocones y trozas recién cortadas de Pinus radiata D. Don: Hylurgus ligniperda (Fabricius), Hylastes ater (Paykull) y Orthotomicus erosus (Wollaston) (Lanfranco et al. 2002a). Actualmente 
las tres especies están distribuidas desde la Región de Valparaíso hasta la de Los $\operatorname{Lagos}^{1}$, en todo el rango de distribución de P. radiata, asociadas a desechos de explotación y plantaciones recién establecidas en sitios postcosecha, con altos niveles poblacionales, sobre todo en la Región del Bío-Bío, donde se concentra el $42 \%$ de las plantaciones de $P$. radiata del país (INFOR 2007), lo que deja de manifiesto el gran potencial de dispersión y establecimiento de dichos escarabajos de corteza.

Estos escolítidos son considerados plagas secundarias, puesto que no provocan mortalidad, no obstante en algunos casos los adultos de $H$. ater e $H$. ligniperda pueden alimentarse de plántulas recién establecidas de $P$. radiata, provocándoles un daño a nivel del cuello y en las raíces, lo que puede ocasionarles la muerte por anillamiento y posterior interrupción del flujo de savia y en ocasiones, facilitar la entrada de hongos patógenos (Parra 1996). El registro en Chile de ataques a plantaciones recién establecidas es muy escaso, probablemente por no haber ocasionado hasta la fecha daños de importancia o por no haber sido registrados, evaluados adecuada y oportunamente, y publicados. En Nueva Zelanda se ha observado el ataque de hasta un $68 \%$ de las plantas producido por $H$. ater en plantaciones establecidas de segunda rotación, lo que muestra el potencial de daño que estos insectos pueden ocasionar y la importancia que tienen los residuos sin manejo postcosecha, que son utilizados por los escarabajos para su alimentación, reproducción y refugio (Lanfranco et al. 2002a, 2004, Reay et al. 2005).

Lanfranco et al. (2004) determinaron que uno de los factores importantes de riesgo que se produce dentro del proceso productivo es el manejo de los desechos. Este manejo en muchas oportunidades no se cumple adecuadamente, lo que se ve reflejado en los altos niveles poblacionales que los escarabajos alcanzan durante la primavera y el verano, épocas en que se realiza la mayoría de las faenas de cosecha.

Dentro de las especies de escarabajos de la corteza en Chile, H. ligniperda es la más abundante, presentando máximas densidades poblacionales durante los meses de septiembre y enero en la Región del Bío-Bío. La situación varía considerablemente en la Región de Los Ríos, en donde H. ligniperda sólo presenta un aumento poblacional en el mes de octubre y con abundancias muy inferiores a las presentadas en la Región del Bío-Bío (Lanfranco et al. 2002b). En este mismo estudio, monitoreando las poblaciones con trampas de embudo, se pudo establecer que H. ligniperda presenta en la zona centro sur de Chile tres a cuatro generaciones al año (aunque solamente se detectan dos máximas poblacionales), en tanto $H$. ater exhibe sólo una máxima poblacional en primavera. Este estudio fue complementado con otro trabajo de campo donde se cortaban trozas a lo largo de todo el año, registrando la

1 Eladio Rojas. Laboratorio de Entomología. Laboratorio Regional Osorno. Servicio Agrícola y Ganadero. Comunicación personal. colonización y observando los estados de ambas especies. Ello permitió constatar el número de generaciones por año y para cada especie. En ambas se encuentran adultos durante todo el año, sin embargo no vuelan en invierno. Machos y hembras reabsorben sus músculos alares al ingresar a las trozas para usar posteriormente esta energía para construir la cámara nupcial, aparearse y colocar sus huevos (Lanfranco et al. 2001, Ruiz et al. 2003).

Según un estudio del United States Department of Agriculture (USDA 1993), estos escarabajos fueron considerados de riesgo alto $(H$. ligniperda) y moderado $(H$. ater y $O$. erosus) de introducción accidental, así como de establecimiento para Norteamérica. Esto, porque tanto $H$. ligniperda como $H$. ater son potenciales vectores de hongos hacia y dentro de Estados Unidos (Cavey et al. 2000, Mausel et al. 2007). Una situación similar presenta México, siendo cuarentenarias para el país las tres especies de escarabajos de la corteza presentes en Chile ${ }^{2}$.

\section{HYLURGUS, HYLASTES Y ORTHOTOMICUS Y SU RELACIÓN CON HONGOS MANCHADORES Y DE PUDRICIÓN}

Los escarabajos de la corteza pueden vivir en una estrecha asociación con hongos de la familia Ophiostomataceae, generalmente asociados a los géneros Ophiostoma y Ceratocystiopsis entre otros, y a sus estados asexuales o anamórficos como Leptographium, Sporotrix y Pesotum (Wingfield y Gibbs 1991). Estos hongos suelen ser económicamente importantes ya que pueden producir el manchado de la madera (estados sexuales) así como enfermedades, principalmente pudrición de raíces, asociadas a estados asexuados de especies del género Leptographium, las que finalmente pueden producir la muerte del árbol. Ellos pueden ser diseminados hasta el árbol a través de muchas especies de escarabajos de la corteza (Zhou et al. 2001).

Dentro de estos hongos, el que representa un mayor riesgo es Leptographium wageneri (pudrición negra de la raíz), que puede estar asociado con especies de los géneros Dendroctonus, Hylastes, Hylurgops e Ips (Coleoptera: Scolytidae) y curculiónidos (Coleoptera: Curculionidae) de los géneros Pissodes y Steremnius (Schweigkofler et al. 2005). Dos especies del género Leptographium, L. lundbergii $(=L$. truncatum) y $L$. procerum, fueron aisladas desde poblaciones de $H$. ligniperda en Nueva Zelanda, lo que hace suponer que su presencia se debe a los escarabajos de la corteza introducidos $H$. ater e H. ligniperda (Cavey et al. 2000). En Chile no se encuentra ninguna de estas especies de Leptographium (Jacobs y Wingfield 2001). La presencia de $L$. serpens en Sudáfrica hace presumir que

2 Juan Pablo López A. Subdepartamento Exportaciones Agrícolas y Forestales. División de Protección Agrícola. Servicio Agrícola y Ganadero. Santiago. Comunicación personal. 
su ingreso al país se hizo a través de $H$. ater (Wingfield y Gibbs 1991).

Tanto los insectos como los hongos pueden tener adaptaciones para transportar y ser transportados respectivamente. Los hongos poseen esporas pegajosas producidas en el ápice o cuello de las fructificaciones que se desarrollan en las galerías y cámaras pupales de los escarabajos. Por otra parte, los insectos pueden llevar las esporas en estructuras especializadas llamadas micangios o sobre el exoesqueleto, antes que ellos emerjan e infesten nuevos árboles (Zhou et al. 2007). Sin embargo, no es necesario que estos escolítidos tengan estructuras especializadas en el transporte de esporas, ya que éstas se adhieren fácilmente al cuerpo del insecto para así diseminarse. Según Beaver (1989), el micangio no siempre es esencial para transmitir exitosamente los hongos; en efecto, estudios afirman que el transporte realizado a través de sistemas relativamente simples parecen ser tan eficientes como el micangio más evolucionado (Francke-Grosmann 1956, Whitney 1982). No obstante esto, tampoco es estrictamente necesario que los escarabajos transporten las esporas, ya que éstas pueden ser diseminadas por el viento y otros agentes variados. Sin embargo, el ingreso de los adultos a las trozas facilita las condiciones para que el hongo entre y se desarrolle en las galerías. Por otra parte, en este tipo de barrenadores de corteza no hay una relación mutualística (insecto-hongo) como ocurre en los escarabajos de ambrosía (Scolytidae y Platypodidae). En una investigación realizada entre los años 2002 y 2003 se comprobó con registros de microscopia electrónica y cortes histológicos que $H$. ligniperda posee micangios primitivos para el transporte de esporas 3,4 .

Las especies de la familia Ophiostomataceae aisladas en distintos países en donde se encuentran establecidas las tres especies de escarabajos de la corteza presentes en Chile, han sido objeto de estudio especialmente en los últimos años (cuadro 1). Se puede apreciar 30 especies de hongos asociadas a estos escolítidos. Para el caso de los ejemplares colectados en Chile, específicamente en la provincia de Valdivia y a los cuales se les aislaron hongos, se encontraron asociados a $H$. ater tres especies del género Ophiostoma: O. huntii, O. galeiformis, O. quercus. Para H. ligniperda se encontraron asociados $O$. ips y Ceratocystiopsis minuta (Zhou et al. 2004). Es por esto que H. ater y H. ligniperda podrían ser considerados vectores no específicos de hongos manchadores y patógenos, debido a la gran cantidad de hongos saprófitos encontrados en sus cuerpos, además de los pertenecientes a la familia Ophiostomataceae. En el caso de Orthotomicus erosus no hay registros de hongos asociados en Chile, pero sí en Sudáfrica y España (Zhou et al. 2001, Romón et al. 2007).

Jimena Muñoz. Forestal Valdivia. Comunicación personal.

Alan Berrymann. Washington State University. Comunicación personal.
Cuadro 1. Hongos de la familia Ophiostomataceae asociados a Hylastes ater e Hylurgus ligniperda.

Ophiostomataceae associated with Hylastes ater and Hylurgus ligniperda.

\begin{tabular}{ll}
\hline \multicolumn{1}{c}{ Hongo } & \multicolumn{1}{c}{ País } \\
\hline & Insecto: Hylastes ater \\
\hline Ophiostoma ips & Nueva Zelanda (1), España (2) \\
O. rectangulosporium & España (2) \\
O. olivaceum & España (2) \\
O. stenoceras & Nueva Zelanda (1), España (2) \\
O. huntii & Nueva Zelanda (1,3, 4), Chile (4) \\
O. galeiformis & Nueva Zelanda (1, 5), Chile (4) \\
O. floccosum & Nueva Zelanda (1), España (2) \\
O. setosum & Nueva Zelanda (1) \\
O. quercus & Nueva Zelanda (1), Chile (4), España (2) \\
O. piliferum & España (2) \\
O. pluriannulatum & España (2) \\
O. piceae & España (2) \\
Leptographium truncatum & Nueva Zelanda (6, 7) \\
L. lundbergii & Nueva Zelanda (7), Reino Unido (8) \\
L. wingfieldii & España (2) \\
L. procerum & Nueva Zelanda (1) \\
L. truncatum & Nueva Zelanda (1), España (2) \\
L. guttulatum & España (2) \\
\hline & Insecto: Hylurgus ligniperda \\
\hline $\begin{array}{l}\text { O. ips } \\
\text { O. galeiformis }\end{array}$ & Chile (4), Sudáfrica (9) \\
O. pluriannulatum & Sudáfrica (9), Chile (4) \\
O. stenoceras & Sudáfrica (9) \\
O. quercus & Sudáfrica (9) \\
Ceratocystiopsis minuta & Sudáfrica (9) \\
L. lundbergii & Chile (4), Sudáfrica (9) \\
L. serpens & Sudáfrica (9) \\
L. guttulatum & Sudáfrica (9) \\
$\begin{array}{l}\text { Pesotum sp. } \\
\text { Sporothrix sp. }\end{array}$ & España (2) \\
Hyalorhinocladiella sp. & Sudáfrica (9) \\
\hline & Sudáfrica (9) \\
Sudrica (9) \\
\hline
\end{tabular}

(1) Reay et al. 2005, (2) Romón et al. 2007, (3) Jacobs et al. 1998, (4) Zhou et al. 2004, (5) Reay et al. 2002, (6) Wingfield y Gibbs 1991, (7) Wingfield et al. 1988, (8) Dowding 1973, (9) Zhou et al. 2001.

Sin duda, uno de los mayores problemas que representa la introducción de $H$. ater y $H$. ligniperda en los países que importan los productos no manufacturados de $P$. radiata es su potencial de establecimiento, sobre todo esta última especie y, junto con ello, la posibilidad de dispersar las esporas por territorios en donde no hay registro de ellos. Estos dos aspectos han sido claves de considerar en los análisis de riesgo de plagas realizados por investigadores tanto de Estados Unidos como de México para establecer medidas de seguridad ante el ingreso de nuestros productos (USDA 1993). Chile está también colaborando en esta tarea, dando cumplimiento al principio de transparencia y de colaboración mutua que se ha declarado. 


\section{LAS EXPORTACIONES DE PRODUCTOS FORESTALES}

El aumento de las exportaciones después de las firmas de los TLC (Tratado de Libre Comercio) con México en agosto de 1999, Estados Unidos en enero de 2004 y Japón en mayo de 2007 trajo consigo un incremento sustancial de las exportaciones forestales, favoreciendo al sector y generando el año 2006 un monto de US\$ 3.890 millones FOB, lo que corresponde a un $6,7 \%$ del total de las exportaciones del país (INFOR 2007, DIRECON 2007). El año 2006 la madera aserrada representó el 10,3\% (2.366,7 miles de metros cúbicos) de las exportaciones forestales del país, donde México y Estados Unidos adquieren en conjunto 980,2 miles de metros cúbicos y Japón 362,6 miles de metros cúbicos, siendo los mercados de destino con mayor demanda. Esto ha incrementado las probabilidades de introducción de especies invasoras desde y hacia Chile. Por lo mismo, los países compradores están demandando cada vez mayores exigencias y restricciones cuarentenarias para la importación de productos primarios.

En numerosos artículos se hace mención del alto riesgo que existe en este intercambio comercial, sobre todo en aquellos productos no manufacturados como trozas y madera aserrada (USDA 1993, Haack y Cavey 2000, Eglitis 2001, Tkacz 2002, Haack 2006, Mausel et al. 2007). Es por esto que el USDA Forest Service y el Animal and Plant Health Inspection Service (APHIS) han llevado a cabo una serie de evaluaciones de riesgo de plagas para el movimiento de especies invasoras debido a la importación de productos primarios. El propósito de estas evaluaciones ha sido identificar las especies que puedan ingresar con los productos importados, evaluar sus probabilidades de introducción y establecimiento, así como estimar sus impactos económicos y ambientales, entre otros.

El año 2006 el Servicio Agrícola y Ganadero (SAG), en consenso con los empresarios privados asociados a la Corporación Chilena de la Madera (CORMA), decidió suspender las exportaciones de madera aserrada verde, debido a la presencia de adultos de $H$. ligniperda en algunos embarques, hasta que se definiera un nuevo modelo de certificación que garantizara un envío seguro desde el punto de vista fitosanitario. A mediados de 2007 se reanudó el envío de maderas aserradas verdes al mercado mexicano. A la fecha se han exportado cuatro embarques denominados "de prueba" porque no son a escala comercial. La evaluación ha sido satisfactoria, el mercado mexicano recuperó las confianzas y próximamente comenzarán las exportaciones con volúmenes importantes ${ }^{5}$. En este mismo contexto la condición fitosanitaria de las exportaciones forestales chilenas ha demandado la aplicación de un

5 Juan Pablo López A. Subdepartamento Exportaciones Agrícolas y Forestales. División de Protección Agrícola. Servicio Agrícola y Ganadero. Av. Bulnes 140 3er piso. Santiago. Comunicación personal. conjunto de medidas técnicas que contribuya, en forma individual o combinada, a mitigar y disminuir el riesgo de infestación por plagas, que, entre otros efectos, dañan la comercialización de los productos forestales. En julio de 2007, y como una forma de fortalecer la actividad productiva exportadora forestal del país, CORMA Bío-Bío creó un sistema de gestión que apoyará la sanidad del sector exportador. Para ello se diseñó un sistema de coordinación y apoyo voluntario a la gestión fitosanitaria de las actividades exportadoras del sector forestal (FITEX) y cuya implementación estará destinada a dar seguridad a los productos forestales de exportación mediante la identificación de riesgos ligados a plagas cuarentenarias y a la adopción de medidas de mitigación eficaces, eficientes y verificables, desde el establecimiento de la plantación hasta el envío de productos a destino (CORMA 2007, CIFAG 2007).

Como complemento a este sistema de control de gestión y con el objetivo de evitar que las maderas que se exportan se contaminen en los puertos, a fines del año 2007 se implementó una zona de resguardo fitosanitario para maderas de exportación en la Región del Bío-Bío. Estas zonas son áreas aisladas donde se inspeccionan, resguardan y consolidan las cargas después de ser aprobadas por los inspectores del SAG, las que posteriormente se exportan principalmente a México. En estos lugares se inspecciona la madera en condiciones de bioseguridad, lo que permite asegurar que el producto no se infeste con escolítidos u otros insectos, evitando así la fumigación preventiva. Existen ya cuatro zonas de resguardo fitosanitario, tres en Talcahuano (provincia de Concepción) y una en Trehuaco (provincia de Nuble), las que contribuyen a asegurar la cadena fitosanitaria de los productos exportados (CORMA 2007).

Complementariamente, se hace necesario tomar medidas de mitigación frente al actual escenario. Es por esto que, a través del Fondo para el Mejoramiento del Patrimonio Fito y Zoosanitario (Fondo SAG), el Instituto de Investigaciones Agropecuarias (INIA) en conjunto con la Controladora de Plagas Forestales (CPF S.A.) comenzarán este año el proyecto "Desarrollo de un sistema de predicción de poblaciones y programa de control biológico de escarabajos de corteza (Hylurgus ligniperda e Hylastes ater) asociados a Pinus radiata". Este proyecto se centrará en el control biológico de los escarabajos de la corteza para reducir los niveles poblacionales, disminuyendo así la infestación de maderas en bruto y elaboradas para exportación. Asimismo, existe la posibilidad de que países importadores de maderas chilenas establezcan medidas fitosanitarias adicionales a las ya existentes.

Las medidas comprometidas y que deben implementarse, así como nuevas iniciativas que se están generando tanto en el sector público como en el privado, se anticipan o responden a las exigencias que los mercados de destino de las exportaciones forestales imponen, y se estima que le hacen bien al país. Se debieran abordar con mayor énfasis 
los problemas de plagas a través de sistemas de protección integral en todas las etapas del proceso productivo, desde el bosque hasta el puerto de destino de los productos, que sean confiables y que aseguren la ausencia de plagas y enfermedades, dando cumplimiento a las normas sanitarias del país importador con todas sus exigencias. De esta forma se incrementaría el prestigio de las exportaciones y se favorecería la competitividad del país en los mercados externos; de lo contrario, la detección de plagas en los productos determinará sanciones legales y económicas con la consiguiente pérdida de mercados.

En síntesis y tras la entrega y análisis de los principales aspectos relacionados con los escarabajos de la corteza, los hongos manchadores y de pudrición que pueden ser transportados por estas especies y la importancia de considerar aspectos técnicos y de gestión en la cadena de valor de los productos primarios de exportación, desde viveros hasta el destino final, se consideran altamente valorables acciones que se han ido desarrollando e implementando para captar y mantener nuestros mercados externos. La tarea conjunta de CORMA, SAG e investigadores nacionales y extranjeros ha permitido llegar a destino con productos de alta calidad, reduciendo el riesgo fitosanitario.

Por su parte, las empresas productoras deben aplicar buenas prácticas silviculturales que contribuyan a este fin, existiendo aún tareas pendientes en el manejo forestal, manejo de desechos, momentos adecuados para plantar, ralear, cosechar, transportar, acopiar e inspeccionar los productos. Mantener los bosques sanos mediante un adecuado manejo, que incluya el manejo integrado de plagas, evitaría muchos de los costos asociados para proteger los productos desde su manufactura a la recepción en los países importadores.

\section{REFERENCIAS}

Beaver R. 1989. Insect-fungus relationships in the bark and ambrosia beetle. In Wilding N, N Collins, P Hammond, J Webber eds. Insect-fungus interactions, England. p. 121-137.

Cavey J, S Passoa y E Hoebeke. 2000. New Introduction: The Redhaired Bark Beetle, Hylurgus ligniperda Fabricius (Coleoptera: Scolytidae). Pest Alert. USDA Forest Service. 2 p.

CIFAG (Colegio de Ingenieros Forestales de Chile, CL). 2007. Panorama Forestal CIF. $N^{\circ} 79$, julio 2007. Consultado 11 ene. 2008. Disponible en http://www.cifag.cl/_file/ file_161_panorama_forestal_julio2007.pdf

CORMA (Corporación Chilena de la Madera, CL). 2007. Sistema Fitosanitario (FITEX). Consultado 4 ene. 2008 Disponible en http://www.cormabiobio.cl/6accionar/departamentos/ intranet/fitex.htm

DIRECON (Dirección General de Relaciones Económicas, CL). 2007. Dirección General de Relaciones Económicas Internacionales. Consultado 20 dic. 2007. Disponible en http://www.direcon.cl

Dowding P. 1973. Effects of felling time and insecticide treatment on the inter-relationships of fungi and arthropods in pine logs. Oikos 24: 422-429.
Eglitis A. 2001. Pest Report. Hylurgus ligniperda. USDA Forest Service. Consultado 20 dic. 2007. Disponible en http://www. spfnic.fs.fed.us/exfor/data/pestreports.cfm?pestidval=59\&lang display=english

Francke-Grosmann H. 1956. Hautdrüsen als träger der pilzsymbiose bei ambrosiakäfern. Zeitschrift fuer Morphologie und Oekologie der Tiere 45: 275-308.

Haack R. 2006. Exotic bark and wood-boring Coleoptera in the United States: recent establishments and interceptions. Canadian Journal of Forest Research 36: 269-288.

Haack R, J Cavey. 2000. Insects intercepted on solid wood packing material at United States ports of entry: 1985-1998. In Proceedings International Conference on Quarentine Pests for the Forestry Sector and their Effects on Foreign Trade. 27-28 junio 2000, Concepción, Chile. CORMA. 16 p.

INFOR (Instituto Forestal, CL). 2007. Estadísticas Forestales Chilenas 2006. 163 p. (Boletín Estadístico 117).

Jacobs K, M Wingfield, B Wingfield, Y Yamaoka. 1998. Comparison of Ophiostoma huntii and O. europhioides and description of $O$. aenigmaticum sp. nov. Mycological Research 102: 289-294.

Jacobs K, MJ Wingfield. 2001. Leptographium Species: tree pathogens, insect associates, and agents of Blue-Stain. APS Press. The American Phytopathological Society. St. Paul MN USA. 224 p.

Lanfranco D, S Ide, H Peredo. 2004. An analysis of health risk reduction in Chilean primary forest products for export. Forestry 77(3): 193-203.

Lanfranco D, S Ide, C Ruiz, H Peredo, I Vives. 2001. Razón sexual de Hylurgus ligniperda (F.), Hylastes ater (Paykull) y Gnathotrupes spp. (Coleoptera: Scolytidae). Bosque 22(2): 85-88.

Lanfranco D, S Ide, C Ruiz, H Peredo, I Vives. 2002a. Agentes entomopatógenos asociados a productos primarios de exportación. Valdivia, Chile. Editorial El Kultrún. 87 p.

Lanfranco D, S Ide, C Ruiz, H Peredo, I Vives. 2002b. Escarabajos de la corteza presentes en las plantaciones de Pinus radiata en Chile. Bosque 23(1): 101-109.

Mausel D, R Gara, D Lanfranco, C Ruiz, S Ide, R Azat. 2007. The introduced bark beetles Hylurgus ligniperda and Hylastes ater (Coleoptera: Scolytidae) in Chile: seasonal flight and effect of Pinus radiata log placement on colonization. Canadian Journal of Forest Research 37: 156-169.

Parra P. 1996. Estudio de los antecedentes biológicos y patrones de ataque en Pinus radiata D. Don de Hylurgus ligniperda (F.) (Coleoptera: Scolytidae) en la zona de Concepción, VIII Región. Tesis Ingeniero Forestal. Concepción, Chile. Facultad de Ciencias Forestales. Universidad de Concepción. 57 p.

Reay S, P Walsh, A Ramb, R Farrell. 2002. The invasion of Pinus radiata seedlings by sapstain fungi, following attack by the Black Pine Bark Beetle, Hylastes ater (Coleoptera: Scolytidae). Forest Ecology and Management 165: 47-56.

Reay S, J Thwaites, R Farrell. 2005. A survey of Ophiostoma species vectored by Hylastes ater to pine seedlings. New Zealand Forest Pathology 35(2): 105-113.

Romón P, X Zhou, J Iturrondobeitia, M Wingfield, A Goldarazena. 2007. Ophiostoma species (Ascomycetes: Ophiostomatales) associated with bark beetles (Coleoptera: Scolytidae) colonizing Pinus radiata in northern Spain. Canadian Journal of Microbiology 53: 756-767. 
Ruiz C, D Lanfranco, D Mausel, B Gara. 2003. Una contribución al conocimiento de los músculos de vuelo de Hylurgus ligniperda (Coleoptera: Scolytidae). Revista Chilena de Entomología 29: 39-42.

Schweigkofler W, W Otrosina, S Smith, D Cluck, K Maeda, K Peay, M Garbelotto. 2005. Detection and quantification of Leptographium wageneri, the cause of black-stain root disease, from bark beetles (Coleoptera: Scolytidae) in Northern California using regular and real-time PCR. Canadian Journal of Forest Research 35: 1798-1808.

Tkacz B. 2002. Pest risks associated with importing wood to the United States. Canadian Journal of Plant Pathology 24: 111-116.

USDA (United States Department of Agriculture, US). 1993. Pest risk assessment of the importation of Pinus radiata, Nothofagus dombeyi, and Laurelia philippiana logs from Chile. Miscellaneous Publication No 1517. 249 p.

Wingfield M, J Gibbs. 1991. Leptographium and Graphium species associated with pine-infesting bark beetles in England. Mycological Research 95: 1257-1260.
Wingfield M, P Capretti, M Mackenzie. 1988. Leptographium spp. as root pathogens of conifers: an international perspective. In Harrington T, F Cobb eds. Leptographium Root Diseases of Conifers. American Phytopathological Society, St Paul. p. 113-128.

Whitney H. 1982. Relationships between bark beetle and symbiotic organisms. In Mitton J, B Sturgeon eds. Bark Beetles in North American conifers. University of Texas Press, Austin. p. 183-211.

Zhou X, Z De Beer, B Wingfield, M Wingfield. 2001. Ophiostomatoid fungi associated with three pine-infesting bark beetles in South Africa. Sydowia 53: 290-300.

Zhou X, Z De Beer, R Ahumada, B Wingfield, M Wingfield. 2004. Ophiostoma and Ceratocystiopsis spp. associated with two pine-infesting bark beetles in Chile. Fungal Diversity 15: 253-266.

Zhou X, T Burgess, W De Beer, F Lieutier, A Yart, K Klepzig, A Carnegie, J Mena, B Wingfield, M Wingfield. 2007. High intercontinental migration rates and population admixture in the sapstain fungus Ophiostoma ips. Molecular Ecology 16: 89-99.

Recibido: 24.01 .08

Aceptado: 29.04.08 